ARTICLE

Received 19 Jul 2013 | Accepted 3 Oct 2013 | Published 1 Nov $2013 \quad$ DOl: 10.1038/ncomms3707

\title{
Flexible and twistable non-volatile memory cell array with all-organic one diode-one resistor architecture
}

Yongsung Ji, ${ }^{1,2}$, David F. Zeigler ${ }^{3}$, Dong Su Lee ${ }^{1}$, Hyejung Choi ${ }^{4}$, Alex K.-Y. Jen ${ }^{3}$, Heung Cho $\mathrm{Ko}^{2} \&$ Tae-Wook Kim ${ }^{1}$

Flexible organic memory devices are one of the integral components for future flexible organic electronics. However, high-density all-organic memory cell arrays on malleable substrates without cross-talk have not been demonstrated because of difficulties in their fabrication and relatively poor performances to date. Here we demonstrate the first flexible all-organic 64-bit memory cell array possessing one diode-one resistor architectures. Our allorganic one diode-one resistor cell exhibits excellent rewritable switching characteristics, even during and after harsh physical stresses. The write-read-erase-read output sequence of the cells perfectly correspond to the external pulse signal regardless of substrate deformation. The one diode-one resistor cell array is clearly addressed at the specified cells and encoded letters based on the standard ASCII character code. Our study on integrated organic memory cell arrays suggests that the all-organic one diode-one resistor cell architecture is suitable for high-density flexible organic memory applications in the future.

\footnotetext{
${ }^{1}$ Soft Innovative Materials Research Center, Institute of Advanced Composite Materials, Korea Institute of Science and Technology, Joellabuk-do 565-905, Korea.

${ }^{2}$ School of Materials Science and Engineering, Gwangju Institute of Science and Technology, Gwangju 500-712, Korea. ${ }^{3}$ Department of Chemistry, University of Washington, Box 352120, Seattle, Washington 98195, USA. ${ }^{4}$ Research and Development Division, SK hynix Inc., Gyeonggi-do 467-701, Korea. Correspondence and requests for materials should be addressed to H.C.K. (email: heungcho@gist.ac.kr) or to T.-W.K. (email: twkim@kist.re.kr).
} 
O rganic-based electronic devices have been intensively investigated in recent decades because of their potential advantages over conventional inorganic electronics, such as their light weight, flexibility, roll-to-roll printing compatibility and the ease of tailoring their optoelectronic properties ${ }^{1-10}$. Recently, various publications have described highly efficient organic solar cells and organic transistors with integrated logic circuits and remarkably low operating voltages ${ }^{11-15}$. Organic memory devices have also been explored for data storage applications ${ }^{2-4,16,17}$. Most research on organic memory devices has focused on the development of new materials or the demonstration of their operation in a simple device structure.

For commercial applications, more research is required to scale down the dimensions of the cell while using advanced cell architectures, effectively pattern the active materials to isolate neighbouring cells in a cross-bar array and fabricate memory cells on flexible substrates ${ }^{2,18-20}$. With regard to minimization of array dimensions, it is well known that a cross-bar array has the highest cell density in a two-dimensional planar device structure $\left(4 F^{2} \text { where } F \text { represents the minimum feature size }\right)^{21}$. However, in the cross-bar array structure, unintended current leakage paths can lead to the misreading of memory states within the cells (commonly called 'cross-talk' between neighbouring cells), and it must be eliminated to achieve precise memory switching operation. In order to solve this cross-talk problem, another functional device should be combined with the memory device. One transistor-one resistor (1T-1R) and one diode-one resistor (1D-1R) can potentially alleviate this cross-talk problem ${ }^{20,22}$. However, these approaches have been based on a hybrid (organic/ inorganic) device structure and could not be printed on flexible substrates or take full advantage of the solution processibility of organic materials. Recently, 1D-1R memory devices with exclusively organic active materials (excluding electrodes) and organic bistable rectifying diodes have been reported ${ }^{23,24}$. However, they were not demonstrated on flexible substrates with a high density of cells, nor did they exhibit essential memory characteristics, such as retention, endurance, in situ pulse response and exhaustive testing, during and after physical stresses. To realize these benchmarks, the active organic materials should be processible at low temperatures to facilitate device fabrication on flexible substrates. In addition, to enhance memory characteristics, the subsequent thin films must be patternable on a desired area and resistant to spin coating of successive layers from organic solutions without eroding. These are essential requisites for not only the integration of organic non-volatile memory arrays on flexible substrates but also the fabrication of organic non-volatile memory cells with advanced architectures and highly reliable performance. Furthermore, both pulse response and precise addressing tests should be carried out on single cells and the entire cell array to examine accurate operation and practical use in organic electronic applications.

In this study, we fabricate ultraviolet-patterned and vertically stacked 64-bit 1D-1R memory cell arrays using poly(3-hexylthiophene) (P3HT) diodes and a mixture of polystyrene (PS) and 6-phenyl- $\mathrm{C}_{61}$ butyric acid methyl ester (PCBM) for the memory components on polyethylene naphthalate (PEN) flexible substrates at low temperature. The memory and diode devices were patterned using a photoactive crosslinker (bis-perfluorobenzoazide: Bis-FB- $\mathrm{N}_{3}$ ) to covalently link the PS and P3HT side chains via nitrene-based $\mathrm{N}-\mathrm{H}$ insertion reactions, which confers solvent resistance to the active layers ${ }^{25}$. Each individual memory and diode device in the integrated memory cell work without malfunction. The 64-bit 1D-1R memory cell arrays also show stable memory characteristics $\left(I_{\mathrm{ON}} / I_{\mathrm{OFF}}\right.$ ratio $\left.\sim 10^{3}\right)$ and reliable retention times $\left(10^{4} \mathrm{~s}\right)$ and endurance cycles $\left(10^{2}\right.$ cycles $)$ after substrate deformation, which is likely to happen during practical use. In addition, the issue of undesired cross-talk between neighbouring cells is solved by integrating the organic diodes directly on top of the organic memory cells. No cross-talk is observed after various mechanical deformations of the flexible substrate. Even during bending deformation, the 1D-1R memory cells are found to respond perfectly to input voltage pulses. Moreover, specific letters are encoded with the 64-bit memory cell array based on the standard ASCII character code.

\section{Results}

Flexible all-organic $1 D-1 R$ cell array. Figure 1 depicts the structure and materials employed in the flexible memory cell array integrated in a vertically stacked 64 -bit $1 \mathrm{D}-1 \mathrm{R}$ architecture developed in this work. Typically, multi-layer organic frameworks experience poor performance or device failure because of erosion during layer-by-layer processing. To avoid this erosion, Bis-FB$\mathrm{N}_{3}$ was used to crosslink the alkyl chains in P3HT thin films via light-catalysed N-H insertion reactions ${ }^{25}$. The Bis-FB- $\mathrm{N}_{3}$ acts as a negative photoresistor responding to $254 \mathrm{~nm}$ ultraviolet light and allows patterning of common alkylated organic materials ${ }^{23,25}$. Crosslinking with Bis-FB- $\mathrm{N}_{3}$ imbues the organic layer with a robust resistance against common organic solvents used for spin coating. In addition, Bis-FB- $\mathrm{N}_{3}$ is one of the few crosslinkers that are compatible with temperature-sensitive plastic substrates. In our previous work, an ultraviolet patternable polyimide (PI) and PCBM blending solution was chosen as a memory component on a rigid substrate, because the PI has good chemical robustness after imidization ${ }^{23}$. Unfortunately, the imidization temperature of $\mathrm{PI}$ is known as over $250^{\circ} \mathrm{C}$ and this issue allows limited use of PI on plastic substrate. Most existing crosslinkers require high temperatures to initiate the crosslinking reaction, which destroys plastic substrates. However, the use of a light-activated crosslinker enables low-temperature processing conditions $\left(\sim 90^{\circ} \mathrm{C}\right)$, which plastic substrates can withstand ${ }^{26,27}$. This approach might be a more efficient way to process multiple organic-soluble layers than use of orthogonal solvents ${ }^{28}$. Figure $1 \mathrm{a}, \mathrm{b}$ shows an illustration and optical image of an as-fabricated all-organic 64-bit 1D-1R memory cell array on a PEN substrate. The entire integrated structure of the $1 \mathrm{D}-1 \mathrm{R}$ cell is (bottom) $\mathrm{PEN} / \mathrm{Al} / \mathrm{PS}: \mathrm{PCBM} / \mathrm{Al} /$ $\mathrm{P} 3 \mathrm{HT} / \mathrm{Au} / \mathrm{Al}$ (top), as shown in Fig. 1c. A P3HT/Bis-FB-N solution was chosen as the diode material (Fig. 1d), and a PS/ $\mathrm{PCBM} / \mathrm{Bis}-\mathrm{FB}-\mathrm{N}_{3}$ mixture was used for the unipolar memory component (Fig. 1e).

To determine whether each device element worked properly in the vertically stacked architecture, an electrical characterization was performed as shown in Fig. 2. Figure 2a shows a currentvoltage $(I-V)$ graph of a ultraviolet-patterned memory cell $(\mathrm{Al} /$ PS:PCBM/Al). It was turned on (set) at $3.5 \mathrm{~V}$ and completely turned off (reset) at $10 \mathrm{~V}$, exhibiting typical unipolar switching behaviour. The memory switching seems to result from the polarization of the PCBM clusters and generation of a strong electric field between adjacent clusters ${ }^{29}$. The $I_{\mathrm{ON}} / I_{\mathrm{OFF}}$ ratio of the devices was greater than $10^{3}$ (at $\left.1.5 \mathrm{~V}\right)$. Figure $2 \mathrm{~b}$ depicts a rectification curve of an integrated $\mathrm{P} 3 \mathrm{HT}$ schottky diode (Al/ $\mathrm{P} 3 \mathrm{HT} / \mathrm{Au}$ ). It exhibits the expected rectification behaviour with a high-rectification ratio of $10^{5}$.

This P3HT diode prevents cross-talk between cells caused by parasitic current paths in a cross-bar array architecture ${ }^{2,18}$. To eliminate the cross-talk problems associated with memory-only (1R) cell arrays, a P3HT diode is vertically integrated onto each memory cell by spin coating a P3HT film over the memory cells, then using a shadow mask to ultraviolet pattern the diodes directly on top of each memory cell, as shown in the inset of Fig. 2c. Unlike the $I-V$ curve of $1 \mathrm{R}$ cells (Fig. $2 \mathrm{a}$ ), the memory cells with a vertically integrated organic diode (1D-1R cell) 


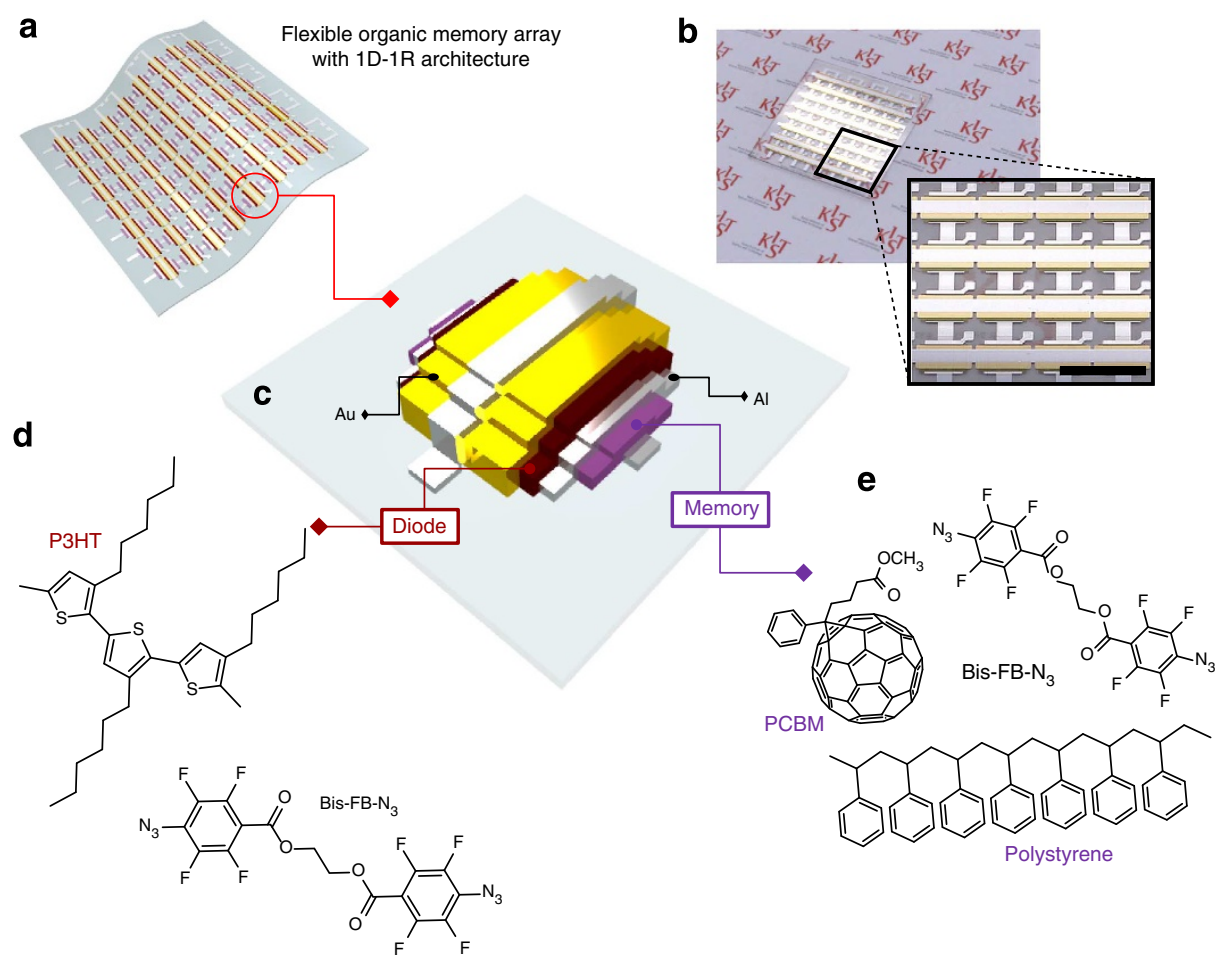

Figure 1 | Flexible all-organic 1D-1R cell. (a) Illustration of as-fabricated 1D-1R organic resistive memory cell array on a flexible PEN substrate and (b) optical image. Scale bar, $5 \mathrm{~mm}$. (c) Schematic of unit cell of 1D-1R cell (AI/PS:PCBM/AI/P3HT/Au/AI). (d) Chemical structure of P3HT and Bis-FB- $\mathrm{N}_{3}$ for ultraviolet patternable diode layer. (e) Chemical structure of PS, PCBM and Bis-FB- $\mathrm{N}_{3}$ for ultraviolet patterable memory layer.
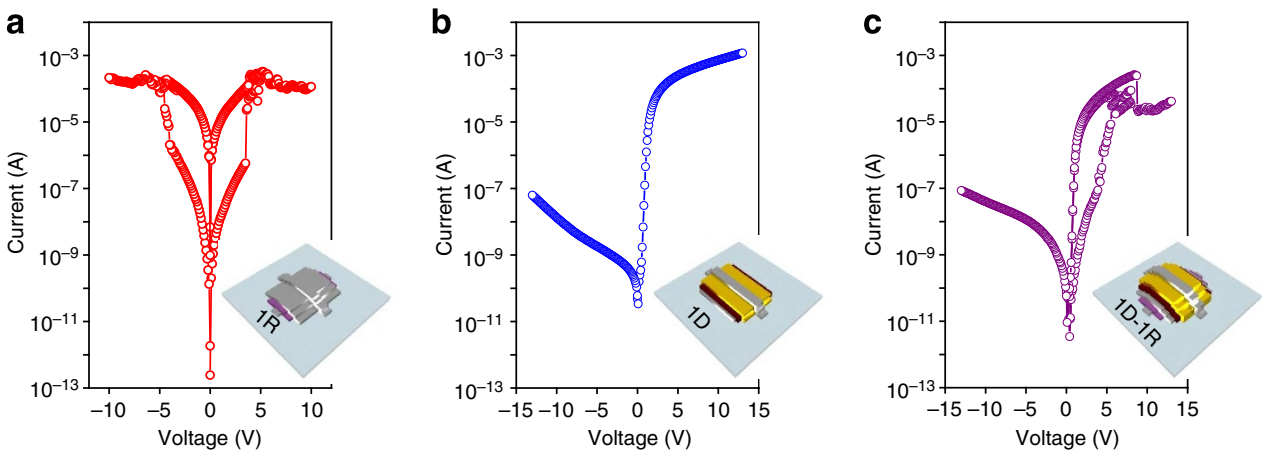

Figure 2 | Memory characteristics of $\mathbf{1 R}$ and $\mathbf{1 D}$ and $\mathbf{1 D}-\mathbf{1 R}$ cell. $I-V$ curve of (a) memory-only cell (1R cell in patterned 1R cell array), (b) diode-only cell (1D cell in patterned 1D cell array) and (c) vertically integrated 1D-1R cell in patterned 1D-1R cell array.

retained their inherent memory characteristics in the forward bias regime because of the ohmic contact between the Au electrode (work function: $5.1 \mathrm{eV}$ ) and P3HT diode (HOMO: $4.9 \mathrm{eV}$ ). On the other hand, the memory cells showed limited current flow in the reverse bias regime because of the Schottky barrier between the $\mathrm{Al}$ electrode (work function: $4.1 \mathrm{eV}$ ) and $\mathrm{P} 3 \mathrm{HT}$ diode (HOMO: $4.9 \mathrm{eV}$ ). For this reason, the $\mathrm{Al}$ middle electrodes were inserted between the memory and diode. The all-organic flexible $1 \mathrm{D}-1 \mathrm{R}$ memory cell was set around $4 \mathrm{~V}$ and completely reset at $13 \mathrm{~V}$. The relatively high operation voltage compared with the memory-only cell might be due to series resistance between the memory and diode components. The electrical properties of the flexible 1D-1R memory cells were characterized using Keithley 4200-SCS and Hewlett and Packard 4145B semiconductor parameter analyser in a $\mathrm{N}_{2}$-filled glove box.
Various mechanical distortions. In order to investigate how the 64-bit all-organic flexible 1D-1R memory cell array behaved under various physical stresses, we first performed electrical characterization of the flexible $1 \mathrm{D}-1 \mathrm{R}$ cells while they were bent (Fig. 3a,b). The PEN substrate $\left(30.3 \times 30.3 \mathrm{~mm}^{2}\right)$ holding the 1D-1R cell was bent from flat to a radius of curvature $(r)$ of $\sim 13 \mathrm{~mm}$ (strain of $0.48 \%$ ) using a home-made holder made from vernier calipers. The detailed information on bending method and estimation of strain are shown in Supplementary Fig. S1 (refs 30-32). As shown as Fig. 3a, the memory device was completely functional, even after bending (strain of $0.48 \%$ ).

For more realistic applications of our all-organic flexible 1D$1 \mathrm{R}$ cell, we did in situ current measurements at the ON, OFF and leakage currents of the organic diode while the flexible substrate was gradually bent. After being turned $\mathrm{ON}$, the ON state current 
a

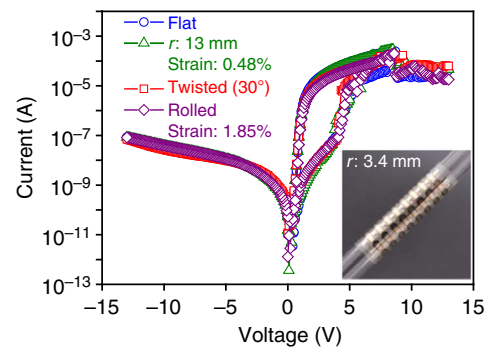

C

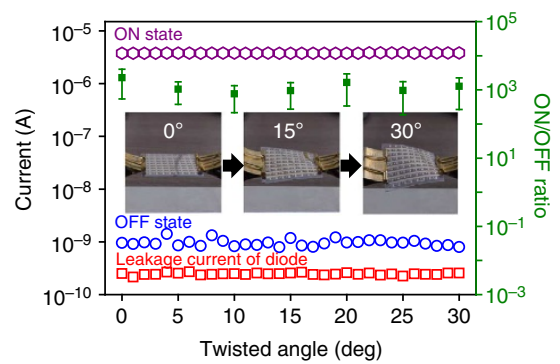

b

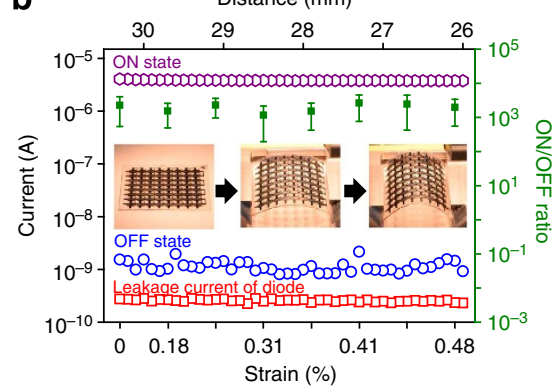

d

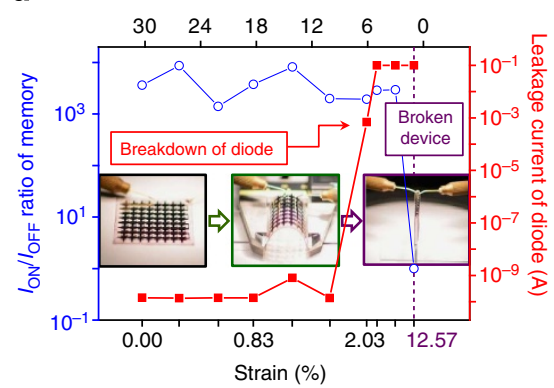

Figure 3 | Memory characteristics of flexible 1D-1R cell. (a) I-V curve of 1D-1R cell before, after bending, twisted and rolled. (b) I-V characteristic of 1D-1R cell under gradual bending and statistical $I_{\mathrm{ON}} / \mathrm{I}_{\mathrm{OFF}}$ ratio distribution of $1 \mathrm{D}-1 \mathrm{R}$ cell under gradual bending. (c) $I-V$ characteristic of $1 \mathrm{D}-1 \mathrm{R}$ cell and statistical $I_{\mathrm{ON}} / I_{\mathrm{OFF}}$ ratio distribution during gradual twisting. (d) $I_{\mathrm{ON}} / I_{\mathrm{OFF}}$ ratio and leakage current of diode until device malfunction.

value was monitored during gradual bending. Interestingly, it did not show large current variation regardless of bending as shown in Fig. 3b (purple hexagons). Similarly, the OFF state current was monitored after the device was turned off under identical bending conditions. The devices displayed consistent OFF current values, as shown in Fig. 3b (blue circles). The 1D-1R cell was measured in the reverse bias regime of the $\mathrm{P} 3 \mathrm{HT}$ diode under the same bending conditions and it retained consistent leakage current values (Fig. 3b, red squares). The ON and OFF currents were read at $1.5 \mathrm{~V}$ (forward bias regime of $1 \mathrm{D}-1 \mathrm{R}$ cell) and the leakage current was read at $-1.5 \mathrm{~V}$ (reverse bias regime of the $1 \mathrm{D}-1 \mathrm{R}$ cell). Regardless of the magnitude or change of the bending radius (increasing strain), the $1 \mathrm{D}-1 \mathrm{R}$ cell retained function of the memory and diode components (resistive change or rectification).

To examine the operational uniformity of the device, $I_{\mathrm{ON}} / I_{\mathrm{OFF}}$ ratios were statistically analysed on 40 working cells under gradual bending conditions. The devices showed high $I_{\mathrm{ON}} / I_{\mathrm{OFF}}$ ratio $\left(\sim 10^{3}\right)$ from flat to bending with strain of $0.48 \%$ (Fig. $3 \mathrm{~b}$ ) and high $I_{\mathrm{ON}} / I_{\mathrm{OFF}}$ ratios $\left(\sim 10^{3}\right)$ during $10^{4}$ times bending cycles (Supplementary Fig. S2a). One bending cycle means the device was bent to strain of $0.48 \%$ and subsequently relaxed to flat. There were negligible $I_{\mathrm{ON}} / I_{\mathrm{OFF}}$ ratio fluctuations, showing a consistent $I_{\mathrm{ON}} / I_{\mathrm{OFF}}$ ratio of $\sim 10^{3}$. The retention test was also performed on cells with increased bending cycle times. The retention time of the memory devices was stable and reliable, even up to $10^{4}$ bending cycles over $10^{4} \mathrm{~s}$ (Supplementary Fig. S2b).

In addition to the bending test, the memory cell array was distorted in other ways. As shown in Fig. 3a,c, we twisted the flexible $1 \mathrm{D}-1 \mathrm{R}$ memory cell array using home-made equipment and measured twisting angles with a protractor (Supplementary Fig. S3). Interestingly, there was not a significant change in $I-V$ curves before and after stressing the substrates at the maximum twist angle $\left(30^{\circ}\right)$. The $1 \mathrm{D}-1 \mathrm{R}$ cell current values were measured in the forward (ON, OFF) and reverse (leakage current of diode) regimes to evaluate whether the flexible $1 \mathrm{D}-1 \mathrm{R}$ devices could withstand gradual twisting stresses. They showed consistent current (ON, OFF and diode leakage) as shown in Fig. 3c, regardless of the twisting angle. The $I_{\mathrm{ON}} / I_{\mathrm{OFF}}$ ratios were statistically analysed on 40 working cells under gradual twisting. They kept their $I_{\mathrm{ON}} / I_{\mathrm{OFF}}$ ratios of $\sim 10^{3}$ from flat to $30^{\circ}$ (Fig. $3 \mathrm{c}$ ). The 1D-1R cell showed stable retention characteristics for more than $10^{4} \mathrm{~s}$, even while the device was twisted by $30^{\circ}$ (Supplementary Fig. S2c). Envisioning an extreme stress condition, the cell array was rolled on a glass pipet with a radius of $3.4 \mathrm{~mm}$ (strain of $1.85 \%$ ) as shown in the inset of Fig. 3a. Despite the small bending radius, the $1 \mathrm{D}-1 \mathrm{R}$ cell worked properly and showed stable retention characteristics over $10^{4} \mathrm{~s}$ (Supplementary Fig. S2c). The detailed descriptions on roll method are illustrated in Supplementary Fig. S4. These results indicate that the allorganic flexible $1 \mathrm{D}-1 \mathrm{R}$ cells have robust operation and reliable electrical properties even under harsh substrate physical deformation. Furthermore, we bent the device more severely to figure out the 1D-1R cell failure during bending condition. The diode (1D) showed malfunction of rectification $\left(10^{-4} \mathrm{~A}\right)$ in negative polarity at strain of $2.03 \%(r \sim 3.1 \mathrm{~mm})$ as shown in Fig. $3 \mathrm{~d}$. In contrast, the memory (1R) was normally operating showing $\sim 10^{3}$ of $I_{\mathrm{ON}} / I_{\mathrm{OFF}}$ ratio. In order to figure out the malfunction of memory, the device was more bent. The memory was working normally showing $\sim 10^{3}$ of $I_{\mathrm{ON}} / I_{\mathrm{OFF}}$ ratio until strain of $6.28 \%$ $(r \sim 1 \mathrm{~mm})$. However, the memory was broken at strain of $12.57 \%$ $(r \sim 0.5 \mathrm{~mm})$ as shown in Fig. 3d. The detailed information on malfunction of $1 \mathrm{D}$ and $1 \mathrm{R}$ is explained in Supplementary Figs S5 and S6. The strain value of each radius of curvature is listed in Supplementary Table S1.

The pulse-response signal of $1 \mathrm{R}$ and $1 \mathrm{D}-1 \mathrm{R}$ cells was tested to determine the fidelity of the write-read-erase-read (WRER) sequence. There have been negligible reports about this measurement on integrated organic memory devices with advanced architectures and under physical stresses. Figure $4 \mathrm{a}, \mathrm{b}$ shows the results of the WRER test on $1 \mathrm{R}$ and $1 \mathrm{D}-1 \mathrm{R}$ cells. The top graph of Fig. 4a shows the input voltage pulse signal applied to the $1 \mathrm{R}$ cell. The cell was subjected to a $900-\mathrm{ms}$ write pulse at $4 \mathrm{~V}$ and a subsequent 300 -ms erase pulse at $10 \mathrm{~V}$ and was read at a voltage of $2 \mathrm{~V}$. The middle and bottom graphs of Fig. 4a show 
a

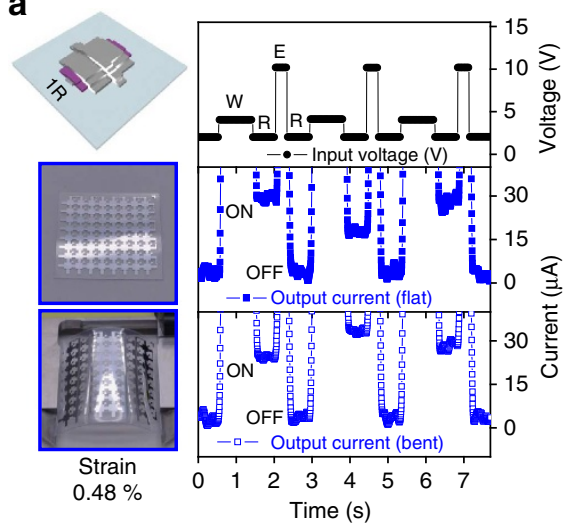

C

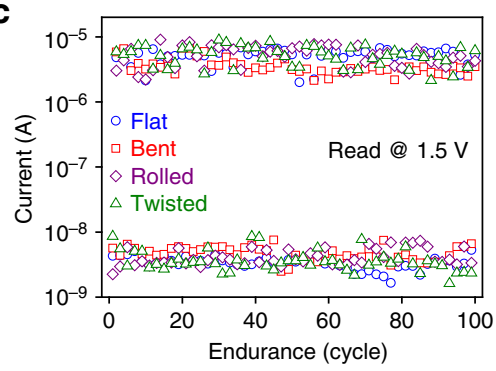

b

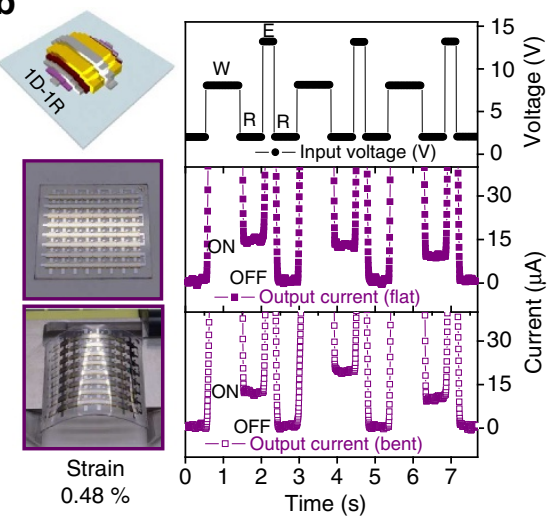

d

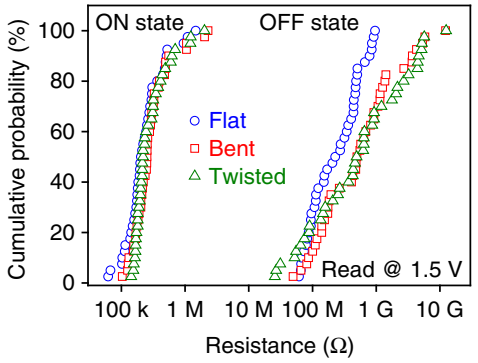

Figure 4 | Pulse response and reliability test of flexible 1D-1R cells. Write-read-erase-read (WRER) cycle test of (a) 1R cell and (b) 1D-1R cell. The graph in the top shows input voltage pulse of (a) 1R cell and (b) 1D-1R cell. The graph in the middle indicates output current response before bending of (a) $1 \mathrm{R}$ cell and (b) 1D-1R cell. The graph in the bottom exhibits output current response after bending (a) 1R cell and (b) 1D-1R cell. (c) Endurance cycles and (d) cumulative probability on various substrate distortion of 1D-1R cell.

the output current response of the $1 \mathrm{R}$ cell before and after bending with strain of $0.48 \%(r \sim 13 \mathrm{~mm})$. The memory cell was completely responsive at input signals at current transitions $(\mathrm{ON}$, OFF). The top of Fig. $4 \mathrm{~b}$ shows the input voltage of the $1 \mathrm{D}-1 \mathrm{R}$ memory cell. A 900 -ms write pulse at $8 \mathrm{~V}$, a 300 -ms erase pulse at $13 \mathrm{~V}$ and a read voltage at $2 \mathrm{~V}$ were used for the response test. Although the operation voltage was increased because of the diode element, the integrated cell exhibited great behaviour, showing outstanding switching current values (ON, OFF state) before and after bending with strain of $0.48 \%(r \sim 13 \mathrm{~mm})$, as shown in the middle and bottom of Fig. $4 \mathrm{~b}$. Endurance cycles and cumulative probability of the flexible $1 \mathrm{D}-1 \mathrm{R}$ device were evaluated when the device was flat, bent, rolled and twisted. As shown in Fig. 4c, the flexible 1D-1R device showed reliable functions without current overlap for both ON and OFF currents even up to $10^{2}$ cycles. In order to check device operational uniformity, the resistance of $\mathrm{ON}$ and OFF current were statistically analysed on 40 working cells under various substrate deformation (bent, twisted) as shown in Fig. 4d. The ON and OFF resistances were perfectly segregated, and the ON current, in particular, showed narrow distribution. The WRER cycles were measured with a two-channel pulse generator (Agillent Technology, 81104A) and a four-channel oscilloscope (Tektronix, DPO 4104B).

Addressing test. In order to figure out the effect of vertically integrated diode, a current addressing test was executed for $1 \mathrm{R}$ devices (memory only), using $1 \mathrm{D}-1 \mathrm{R}$ devices for comparison. As shown in the inset of Fig. 5a, an OFF current (that is, the '0' state) was applied to one cell of the $1 \mathrm{R}$ device. Subsequently, an ON current (that is, the ' 1 ' state) was applied to three adjacent cells. Then, the current was read at each of the four cells at $1.5 \mathrm{~V}$. In the
$1 \mathrm{R}$ device, the ON and OFF currents were not correctly read because of cross-talk between the neighbouring cells (Fig. 5b). Despite programming one cell to an OFF current $\left(\sim 10^{-8} \mathrm{~A}\right)$, all the cells displayed ON currents $\left(\sim 10^{-5} \mathrm{~A}\right)$, as shown in the left histogram of Fig. 5c. The same experiment was carried out on the $1 \mathrm{D}-1 \mathrm{R}$ device as shown as Fig. 5d. Unlike the $1 \mathrm{R}$ device, the $1 \mathrm{D}-1 \mathrm{R}$ device shows precise addressing (Fig. 5e). In the $1 \mathrm{D}-1 \mathrm{R}$ device, the current state of the OFF cell was read correctly. Owing to the vertically integrated diode, the reverse current path (bottom to top electrodes) was blocked because of the Schottky barrier but the forward current path (top to bottom electrodes) was open because of ohmic contact. Thus, the information in the OFF state can be perfectly read as shown in the right histogram of Fig. $5 \mathrm{c}$. Verifying the accurate addressing in the 64-bit 1D-1R memory cell array, we wrote 'KIST' as shown in Fig. 5f. The letters of 'KIST' were programmed based on the ON (1) and OFF (0) currents by the standard ASCII character code in $3 \times 3$ cells (for each letter) among the $8 \times 8$ cells array $1 \mathrm{D}-1 \mathrm{R}$ device.

\section{Discussion}

We fabricate a 64-bit all-organic flexible memory cell array in a 1D-1R architecture with photopatternable organic memory (PS:PCBM) and diode (P3HT) systems. Light-catalysed crosslinking enables low-temperature materials processing, which thereby allows device fabrication on a flexible PEN substrate, resulting in the first fully flexible memory device. These $1 \mathrm{D}-1 \mathrm{R}$ cells showed good electronic behaviour and rewritable switching, even during and after harsh physical stresses. They retain their respective current values $(\mathrm{ON}$, OFF and leakage current of the diode) during gradual substrate deformation without sacrificing their individual electrical properties. The $1 \mathrm{D}-1 \mathrm{R}$ cells show stable retention characteristics over $10^{4} \mathrm{~s}$ even while undergoing 
a

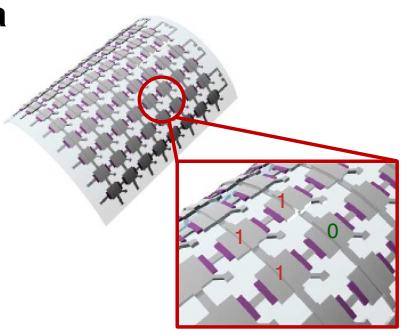

d

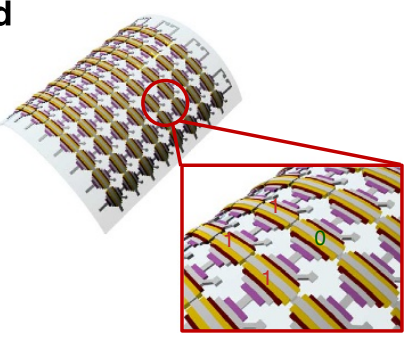

b

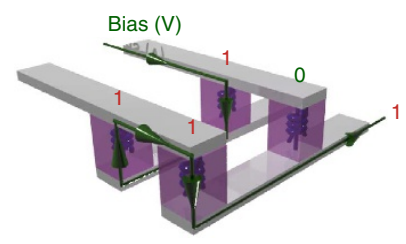

e

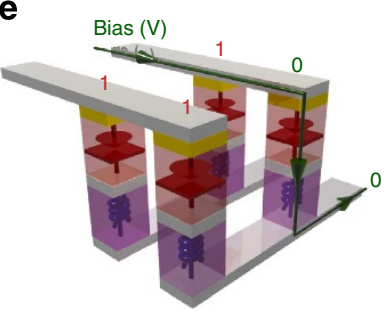

C

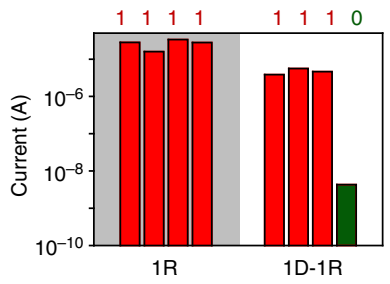

f

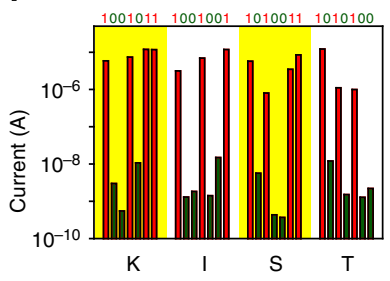

Figure 5 | Addressing test of $\mathbf{1 R}$ and $\mathbf{1 D}-\mathbf{1 R}$ cells. (a) Programmed current states of $1 \mathrm{R}$ cells. (b) Current path without stacked diode (1R cells). (c) Histogram of current states during the reading process of $1 R$ cells and 1D-1R cells. (d) Programmed current states of 1D-1R cells. (e) Current path with stacked diode (1D-1R cells). (f) The output currents histogram exhibiting the letter 'KIST' by the standard ASCII characters within the $8 \times 8$ 1D-1R cells.

substrate distortion up to $10^{4}$ bending cycles. An in situ measurement of the response signal via the external pulse signal with WRER sequences reveals that our $1 \mathrm{D}-1 \mathrm{R}$ cell array is a candidate for practical use in high-density all-organic flexible memory applications. Furthermore, the all-organic flexible $1 \mathrm{D}-1 \mathrm{R}$ cell array successfully avoids cross-talk problems and can write specific letters based on standard ASCII character code, illustrating promising operational performance for practical flexible organic memory applications. Also, the $1 \mathrm{D}-1 \mathrm{R}$ cells show reliable reproducibility and uniformity regardless of mechanical device deformation. Our research represents one of the initial key studies describing the fabrication and characterization of integrated flexible organic electronics exploiting sophisticated architectures.

\section{Methods}

Material preparations. In order to prepare the memory cell active material, PS $(7 \mathrm{mg})$ and PCBM $(1 \mathrm{mg})$ were dissolved into chlorobenzene $(1 \mathrm{ml})$ with Bis-FB- $\mathrm{N}_{3}(5 \mathrm{mg})$ to make the ultraviolet patternable solution. For preparation of the organic diode solution, $\mathrm{P} 3 \mathrm{HT}(55 \mathrm{mg})$ was dissolved in chlorobenzene $(1 \mathrm{ml})$ with Bis-FB- $\mathrm{N}_{3}(5 \mathrm{mg})$.

Fabrication of flexible 1D-1R cell array. A $125-\mu \mathrm{m}$-thick PEN substrate $\left(30.3 \times 30.3 \mathrm{~mm}^{2}\right)$ was prepared for the flexible $1 \mathrm{D}-1 \mathrm{R}$ memory device and cleaned by acetone and isopropyl alcohol. The 70-nm thick $\mathrm{Al}$ bottom electrodes were deposited using a shadow mask by a thermal evaporator with a deposition rate of $5 \AA \mathrm{s}^{-1}$ at a pressure of $\sim 10^{-7}$ torr. The $\mathrm{Al}$ bottom electrodes deposited on the PEN substrate were exposed to ultraviolet-ozone to increase film uniformity ${ }^{33}$. The prepared memory solution was filtered through a $0.45-\mu \mathrm{m}$ polytetrafluoroethylene filter and spin coated onto the substrate at 500 r.p.m. for $5 \mathrm{~s}$ and then at 1,000 r.p.m. for $60 \mathrm{~s}$. After spin coating, the substrate was exposed to ultraviolet $(254 \mathrm{~nm})$ light using a shadow mask for $5 \mathrm{~min}$ to initiate the crosslinking reaction, then rinsed with chlorobenzene at 4,000 r.p.m. for $60 \mathrm{~s}$. The patterned device was annealed at $90{ }^{\circ} \mathrm{C}$ for $10 \mathrm{~min}$ in a $\mathrm{N}_{2}$ filled glove box. As the top electrodes of the memory cells and the bottom electrodes of diode cells, $70 \mathrm{~nm}$ thick $\mathrm{Al}$ middle electrodes were deposited by thermal evaporator using a shadow mask. The prepared diode solution was spin coated at 1,500 r.p.m. for $60 \mathrm{~s}$ and then exposed to ultraviolet $(254 \mathrm{~nm})$ light using a shadow mask for $5 \mathrm{~min}$ to initiate crosslinking then rinsed with chlorobenzene at 4,000 r.p.m. for 60 s. As the top electrodes for the diode, $\mathrm{Au}$ electrodes $(100 \mathrm{~nm})$ were deposited by a thermal evaporator using a shadow mask. For the arrayed 1D-1R device, the Al top electrodes were deposited by thermal evaporator using a shadow mask. The detailed fabrication process information is described in the Supplementary Fig. S7. The thickness of the memory layer was $\sim 70 \mathrm{~nm}$ and the diode layer was $\sim 300 \mathrm{~nm}$ (Supplementary Fig. S8).

\section{References}

1. Forrest, S. R. The path to ubiquitous and low-cost organic electronic appliances on plastic. Nature 428, 911-918 (2004).

2. Ling, Q.-D. et al. Polymer electronic memories: materials, devices and mechanisms. Prog. Polym. Sci. 33, 917-978 (2008).

3. Scott, J. C. \& Bozano, L. D. Nonvolatile memory elements based on organic materials. Adv. Mater. 19, 1452-1463 (2007).

4. Yang, Y., Ouyang, J., Ma, L., Tseng, R. J.-H. \& Chu, C.-W. Electrical switching and bistability in organic/polymeric thin films and memory devices. Adv. Funct. Mater. 16, 1001-1014 (2006).

5. Singh, M., Haverinen, H. M., Dhagat, P. \& Jabbour, G. E. Inkjet printingprocess and its applications. Adv. Mater. 22, 673-685 (2010).

6. Wang, J. et al. Entirely solution-processed write-once-read-many-times memory devices and their operation mechanism. Org. Electron 12, 1271-1274 (2011).

7. Park, H. J., Kang, M.-G., Ahn, S. H. \& Guo, L. J. A facile route to polymer solar cells with optimum morphology readily applicable to a roll-to-roll process without sacrificing high device performance. Adv. Mater. 22, E247-E253 (2010).

8. Kim, J. Y. et al. Efficient tandem polymer solar cells fabricated by all-solution processing. Science 317, 222-225 (2007).

9. Sekitani, T. et al. Stretchable active-matrix organic light-emitting diode display using printable elastic conductors. Nat. Mater. 8, 494-499 (2009).

10. Menard, E. et al. Micro- and nanopatterning techniques for organic electronic and optoelectronic systems. Chem. Rev. 107, 1117-1160 (2007).

11. He, Z. et al. Enhanced power-conversion efficiency in polymer solar cells using an inverted device structure. Nat. Photonics 6, 591-595 (2012).

12. Sekitani, T. et al. Organic nonvolatile memory transistors for flexible sensor arrays. Science 326, 1516-1519 (2009).

13. Acton, O. et al. $\pi-\sigma$-Phosphonic acid organic monolayer/sol-gel hafnium oxide hybrid dielectrics for low-voltage organic transistors. Adv. Mater. 20, 3697-3701 (2008).

14. Cosseddu, P., Lai, S., Barbaro, M. \& Bonfiglio, A. Ultra-low voltage, organic thin film transistors fabricated on plastic substrates by a highly reproducible process. Appl. Phys. Lett. 100, 093305 (2012).

15. Acton, O. et al. Simultaneous modification of bottom-contact electrode and dielectric surfaces for organic thin-film transistors through single-component spin-cast monolayers. Adv. Funct. Mater 21, 1476-1488 (2011).

16. Ouyang, J., Chu, C.-W., Szmanda, C. R., Ma, L. \& Yang, Y. Programmable polymer thin film and non-volatile memory device. Nat. Mater. 3, 918-922 (2004).

17. Ji, Y., Lee, S., Cho, B., Song, S. \& Lee, T. Flexible organic memory devices with multilayer graphene electrodes. ACS Nano 5, 5995-6000 (2011).

18. Cho, B., Song, S., Ji, Y., Kim, T.-W. \& Lee, T. Organic resistive memory devices: performance enhancement, integration, and advanced architectures. Adv. Funct. Mater. 21, 2806-2829 (2011).

19. Kwan, W. L., Tseng, R. J. \& Yang, Y. Multi-layer stackable polymer memory devices. Philos. Trans. R. Soc. A 367, 4159-4167 (2009). 
20. Kim, T.-W. et al. One transistor-one resistor devices for polymer non-volatile memory applications. Adv. Mater. 21, 2497-2500 (2009).

21. Lee, M.-J. et al. A low-temperature-grown oxide diode as a new switch element for high-density, nonvolatile memories. Adv. Mater. 19, 73-76 (2007).

22. Cho, B. et al. Rewritable switching of one diode-one resistor nonvolatile organic memory devices. Adv. Mater. 22, 1228-1232 (2010).

23. Kim, T.-W. et al. All-organic photopatterned one diode-one resistor cell array for advanced organic nonvolatile memory applications. Adv. Mater. 24, 828-833 (2012).

24. Asadi, K., Li, M., Stingelin, N., Blom, P. W. M. \& de Leeuw, D. M. Crossbar memory array of organic bistable rectifying diodes for nonvolatile data storage. Appl. Phys. Lett. 97, 193308 (2010).

25. Liu, L.-H. \& Yan, M. Perfluorophenyl azides: new applications in surface functionalization and nanomaterial synthesis. Acc. Chem. Res. 43, 1434-1443 (2010).

26. Fix, W., Ullmann, A., Ficker, J. \& Clemens, W. Fast polymer integrated circuits. Appl. Phys. Lett. 81, 1735-1737 (2002).

27. Eder, F. et al. Organic electronics on paper. Appl. Phys. Lett. 84, 2673-2675 (2004).

28. Baeg, K.-J., Noh, Y.-Y., Sirringhaus, H. \& Kim, D.-Y. Controllable shifts in threshold voltage of top-gate polymer field-effect transistors for applications in organic nano floating gate memory. Adv. Funct. Mater. 20, 224-230 (2010).

29. Laiho, A. et al. Tuning the electrical switching of polymer/fullerene nanocomposite thin film devices by control of morphology. Appl. Phys. Lett. 93, 203309 (2008).

30. Yun, D. Y., Song, W. S., Kim, T. W., Kim, S. W. \& Kim, S. W. Electrical stabilities and carrier transport mechanisms of flexible organic bistable devices based on CdSe-InP core-shell nanoparticle/polystyrene nanocomposites. Appl. Phys. Lett. 101, 103305 (2012).

31. Kim, S. \& Choi, Y.-K. Resistive switching of aluminum oxide for flexible memory. Appl. Phys. Lett. 92, 223508 (2008).
32. Jang, J., Pan, F., Braam, K. \& Subramanian, V. Resistance switching characteristics of solid electrolyte chalcogenide $\mathrm{Ag}_{2} \mathrm{Se}$ nanoparticles for flexible nonvolatile memory applications. Adv. Mater. 24, 3573-3576 (2012).

33. Verbakel, F. et al. Reproducible resistive switching in nonvolatile organic memories. Appl. Phys. Lett. 91, 192103 (2007).

\section{Acknowledgements}

This work was supported by the Korea Institute of Science and Technology (KIST) Institutional Program and the R\&D program funded by Jeollabuk-do.

\section{Author contributions}

T.-W.K. and H.C.K. planned and supervised the project. T.-W.K. and Y.J. designed and performed the experiments. T.-W.K. and Y.J. analysed and interpreted the data and wrote the manuscript. D.F.Z. synthesized the Bis-FB- $\mathrm{N}_{3}$ and D.S.L assisted in pulseresponse test. H.C.K. and A.K.-Y.J. contributed to discussions throughout the project.

\section{Additional information}

Supplementary Information accompanies this paper at http://www.nature.com/ naturecommunications

Competing financial interests: The authors declare no competing financial interests.

Reprints and permission information is available online at http://npg.nature.com/ reprintsandpermissions/

How to cite this article: Ji, Y. et al. Flexible and twistable non-volatile memory cell array with all-organic one diode-one resistor architecture. Nat. Commun. 4:2707 doi: 10.1038/ ncomms3707 (2013). 